\title{
Practices in the evaluation of potential kidney transplant recipients who are elderly: A survey of U.S. transplant centers
}

\author{
Didier A. Mandelbrot ${ }^{1}$ (D) | Aaron Fleishman ${ }^{2}$ | James R. Rodrigue ${ }^{2}$ (D) | \\ Silas P. Norman ${ }^{3}$ | Milagros Samaniego ${ }^{3}$
}

${ }^{1}$ Department of Medicine, UWHealth, Madiso, WI, USA

${ }^{2}$ The Transplant Institute, Beth Israel Deaconess Medical Center, Boston, MA, USA

${ }^{3}$ Department of Medicine, The University of Michigan, Ann Arbor, MI, USA

\section{Correspondence}

Didier A. Mandelbrot, Department of Medicine, UWHealth, Madiso, WI, USA.

Email: damandel@medicine.wisc.edu

Funding information

This work was supported by an unrestricted research grant from the Virginia Lee Cook Foundation.

\begin{abstract}
Limited data exist regarding the evaluation and selection of older candidates for transplantation. To help guide the development of program protocols and help define research questions in this area, we surveyed U.S. transplant centers regarding their current practices in the evaluation of older kidney transplant candidates. We emailed a 28-question survey to the medical and surgical directors of 190 adult kidney transplant programs in the USA. We received usable responses from 59 programs, a 31.1\% response rate. Most (76.3\%) programs do not have absolute age cutoffs for listing patients, but for the $22.0 \%$ of programs that do have cutoffs, the mean age was 79 , range $70-90$. Nearly one-third (29.2\%) of programs require a minimum life expectancy to list for transplant, reporting a mean of 4.5 years life expectancy, (range 2-10). Programs vary significantly in evaluating candidates living in a nursing home or with cognitive impairments. Practices regarding the evaluation of older transplant candidates vary widely between U.S. programs. Further studies are needed on the impact of age and other comorbidities on transplant outcomes, to help guide decisions on which older patients are most appropriate for transplant listing.

KEYWORDS

assessment, clinical decision-making, organ allocation, patient characteristics, risk, risk stratification, waitlist management
\end{abstract}

\section{1 | INTRODUCTION}

As the U.S. population continues to live longer, and the number of older patients listed for kidney transplantation continues to increase, ${ }^{1,2}$ decisions about which patients to exclude from listing have become increasingly common. Older recipients generally have more cardiovascular and other comorbidities, including cognitive and functional impairments. As a result, most ${ }^{1,3}$ although not all ${ }^{4}$ studies have shown that older recipients have worse graft and patient outcomes than younger recipients. However, many studies have demonstrated that older recipients have better survival with kidney transplantation than with dialysis ${ }^{5-7}$ although depending on patient selection, this is not always the case. ${ }^{8}$ Where exactly to draw the line in accepting candidates for transplantation continues to be challenging. While some predictive models as to which older candidates would benefit have been proposed, ${ }^{9,10}$ a consensus conference in $\mathbf{2 0 1 2}$ on solid organ transplantation in older adults noted how few studies have addressed these issues. ${ }^{11}$

Documenting current practices in the evaluation of older candidates is an important step in guiding the development of program protocols and helping define research questions in this area. To this end, we surveyed U.S. transplant centers regarding their current practices in the evaluation of older kidney transplant candidates.

\section{2 | MATERIALS AND METHODS}

We designed a 28-question web-based survey to obtain information on practices regarding the evaluation of elderly candidates for kidney 
transplantation. We purchased from UNOS a list of medical and surgical directors from all 222 kidney transplant programs, and excluded the 30 pediatric-only programs. Because UNOS does not provide e-mail addresses, we searched the Internet and called transplant centers to obtain the current e-mail addresses of the medical or surgical director. We were unable to obtain email addresses for two programs. We then sent to 190 programs an email describing the study along with a secured hyperlink to complete the online survey. The email was sent three times between $5 / 3 / 13$ and $6 / 19 / 13$, with only programs who had not responded receiving repeat requests to answer. We asked respondents to provide their UNOS program code, so we could track whether multiple responses were received from a particular program. For the two program that had two respondents, we used the medical director's responses in the statistical analyses. We used the RedCap survey manager to send emails and manage responses. All answers were kept anonymous. Survey responses were coded and downloaded into Statistical Package for the Social Sciences Version 19 (IBM Corp., Armonk, NY, USA) for analysis.

Data are expressed as median, mean and SD, or the percentage of centers with specific responses. Survey responses were examined by respondent age (median split), respondent specialty (nephrologist vs. surgeon), program patient volume (median split), and percentage of transplant recipients $\geq 65$ year old (median split). Analyses included $t$-tests for continuous variables, Fisher's exact test for variables with two categories, or a two-tailed chi-squared test for variables with three or more categories. A Bonferroni correction was made for multiple comparisons to determine statistical significance.

\section{3 | RESULTS}

\section{1 | Respondent demographics}

Of the 190 programs surveyed, we received responses from 67 (35.3\%). Six surveys were returned with no answers, and for two programs, both a nephrologist and surgeon returned surveys, so only the nephrologist's answers were used in our analysis. Therefore, our effective response rate was $59 / 190$ or $31.1 \%$. Respondents were $50.9 \%$ nephrologists, $43.4 \%$ surgeons, and $5.7 \%$ other positions. Because the survey questions relate to age, we asked respondents their own age: the median age was 51 , mean $51.5( \pm 8.63)$, and range 36-71. The number of kidney transplants performed in 2012, not including combined organs, was a median 80 , mean $108.5 \pm 77.5$, range 21-350.

The reported percentage of kidney transplant recipients $\geq 65$ years old is shown in Figure 1. The median percentage of recipients $\geq 65$ years old was $20 \%$, mean $23 \pm 11.4 \%$, with a very wide range of older recipients, $3 \%-50 \%$ of transplants. The reported percentage of waitlisted patients $\geq 65$ years old was median $25 \%$, mean $23.9 \pm 9.8 \%$, range $7.5 \%-50 \%$. The slightly higher proportion of patients $\geq 65$ years old on the waitlist compared to those receiving transplants likely reflects the lower likelihood of transplantation for older patients. All UNOS regions were represented among respondents, with \% of total responses $(n=54)$ ranging from $5.6 \%$ from region 3 , to $16.7 \%$ from region 2 .

\section{2 | Practices regarding listing of older patients}

When asked about the transplant program's upper age cutoff for listing a patient for kidney transplantation $(n=59), 76.3 \%(n=45)$ reported having no cutoff and $1.7 \%(n=1)$ reported another policy. The $22.0 \%(n=13)$ of programs with a specific cutoff reported an upper age limit ranging from 70 to 90 years old, with a median of 80 and mean $79 \pm 5.5$ years old. Of the respondents with no overall cutoff for listing, a number of programs reported having age limits for listing patients with specific comorbidities, the most common being heart disease (Figure 2).

We also asked whether programs have a cutoff for listing transplant candidates based on life expectancy without transplant. Of the 59 responders, 50.8\% reported no cutoff policy, but for the remaining $49.2 \%$, the years of life expectancy required to list ranged from 2 to 10 , with a mean of $4.5 \pm 1.7$ and median of 5 . In a separate question about listing candidates expected to only benefit from

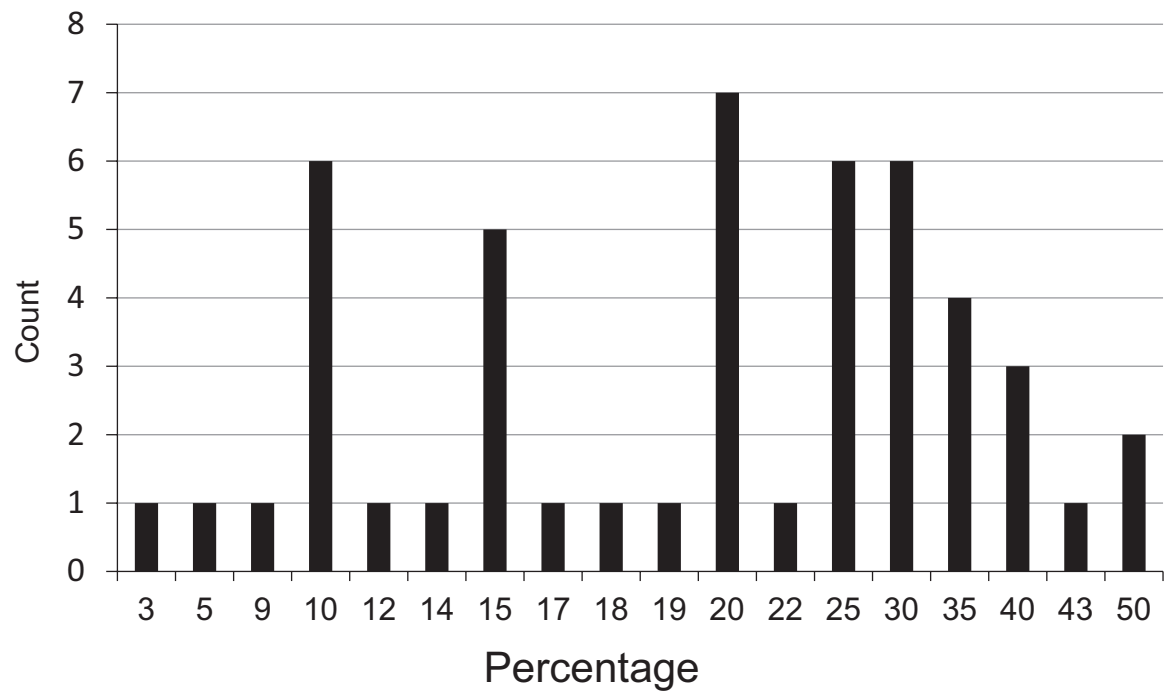

FIGURE 1 What percentage of your kidney transplant recipients are $\geq 65$ years old? $\mathrm{n}=49$ 
FIGURE 2 If your program has no upper age cutoff for listing, does it have an age cutoff that applies to listing potential recipients with specific medical conditions (choose all that apply)? $n=46$

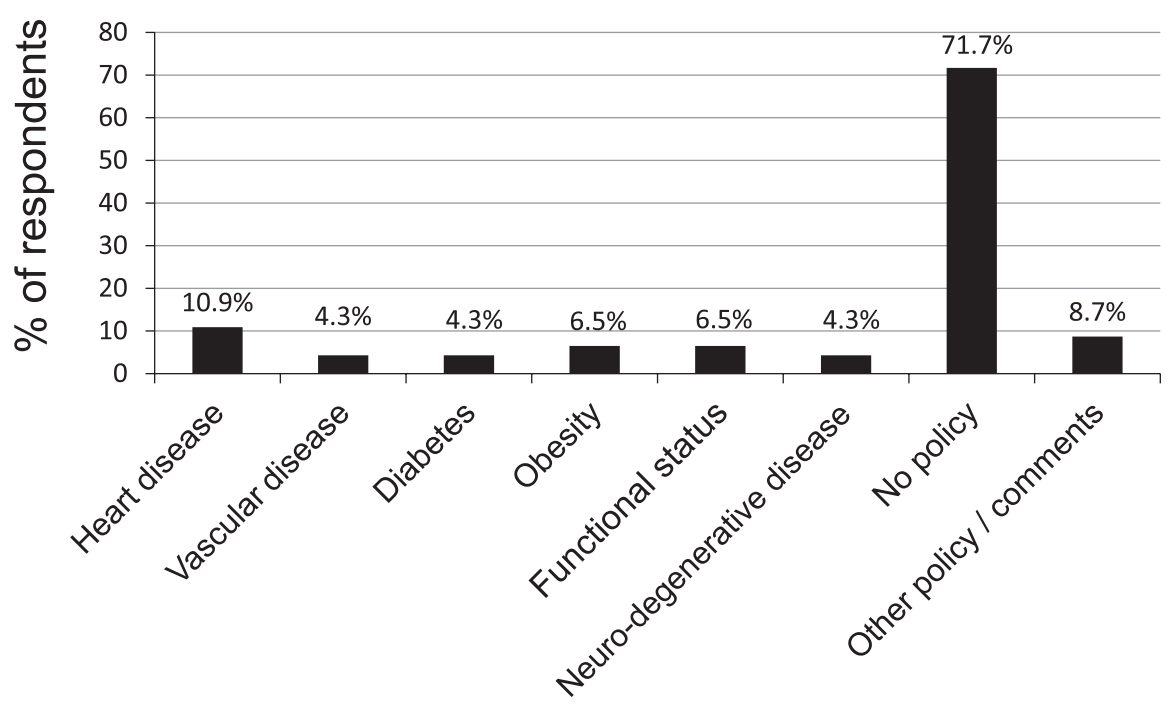

transplantation in terms of quality of life, but not length of life, most ( $61 \%$ of $n=58$ ) reported that they would list such candidates, while $25.4 \%$ would not.

The next series of questions related to impairments sometimes found in older transplant candidates. When asked about listing candidates who live in a nursing home (Figure 3), the most common practice is to exclude them from listing (41.4\%), but the second most common response $(24.1 \%)$ was that nursing home residency is not a factor for acceptance. Other programs would accept candidates based on specific reasons for nursing home residency. Cognitive impairment was not necessarily a reason for exclusion (Figure 4), with the most common response being that candidates would be excluded only if cognitive impairment was accompanied by lack of good supports to ensure compliance (72.9\%). A minority of programs would use cognitive impairment as a listing exclusion only if the impairment was expected to be progressive or if the impairment was "significant." When asked whether a formal cognitive assessment is part of the routine transplant evaluation, $16.9 \%$ of programs reported "always" while $69.5 \%$ answered that a formal assessment is only performed when informal assessments suggests doing so. Only one program (1.7\%) reported performing formal assessments based on an age cutoff ( 75 years old). When programs were asked about accepting candidates with functional impairment (Figure 5), the answers were similar to those about cognitive impairment, with most programs (61\%) excluding candidates only if the candidate also does not have good supports, or the impairment was expected to be progressive (32.2\%). The approach to formal functional assessments is similar to that for formal cognitive assessments, with $25.4 \%$ of programs always performing a functional assessment as part of the transplant evaluation, and $59.3 \%$ doing the functional assessment if informal assessments suggested doing so. Almost all programs have the same psychosocial evaluations of candidates based on age $196.6 \%$ of $n=58)$ and have the same upper age policy based on sex $(96 \%$ of $n=57)$ and race/ethnicity (94.9\% of $n=59)$.

To assess the interaction between living donation and listing of older transplant candidates, we asked programs about upper age cutoffs to accept living donors; $83.5 \%$ of programs do not have an age cutoff for patients to receive a live donor kidney, but of the $13.8 \%$ $(n=8)$ that do have a cutoff, the mean age was $75 \pm 8.5$, range $60-85$. One program (1.7\%) reported that the cutoff depends on the donor relation. When asked whether a nondirected live donor would be accepted for an older recipient, $78.6 \%$ of 58 respondents said yes, while $8.9 \%$ would not accept a nondirected donor above an age cutoff ranging from 60 to 70 , mean $68 \pm 4.5$.
FIGURE 3 Does your program accept potential recipients who live in a nursing home? $(n=58)$

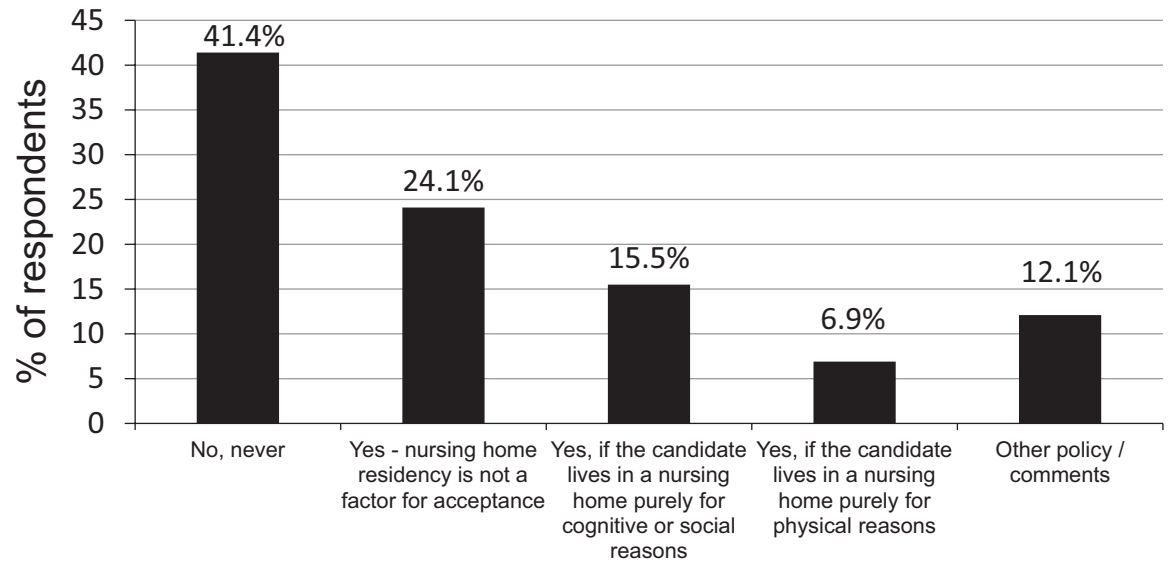




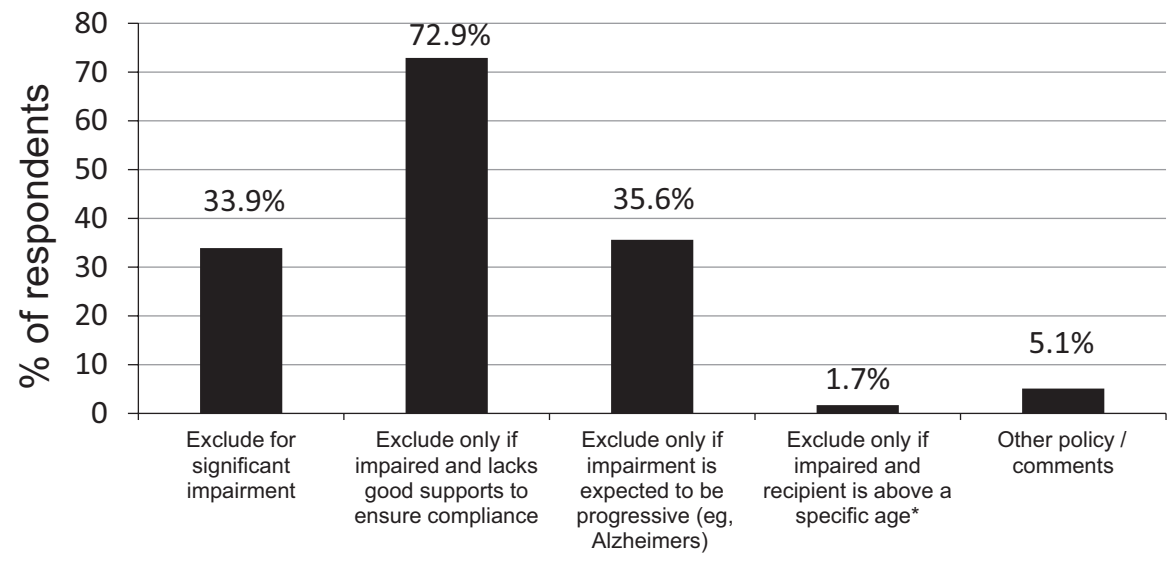

*The one response stated their age cutoff was 70
FIGURE 4 What is your program's practice regarding potential recipients with cognitive impairment (choose all that apply)? n = 59

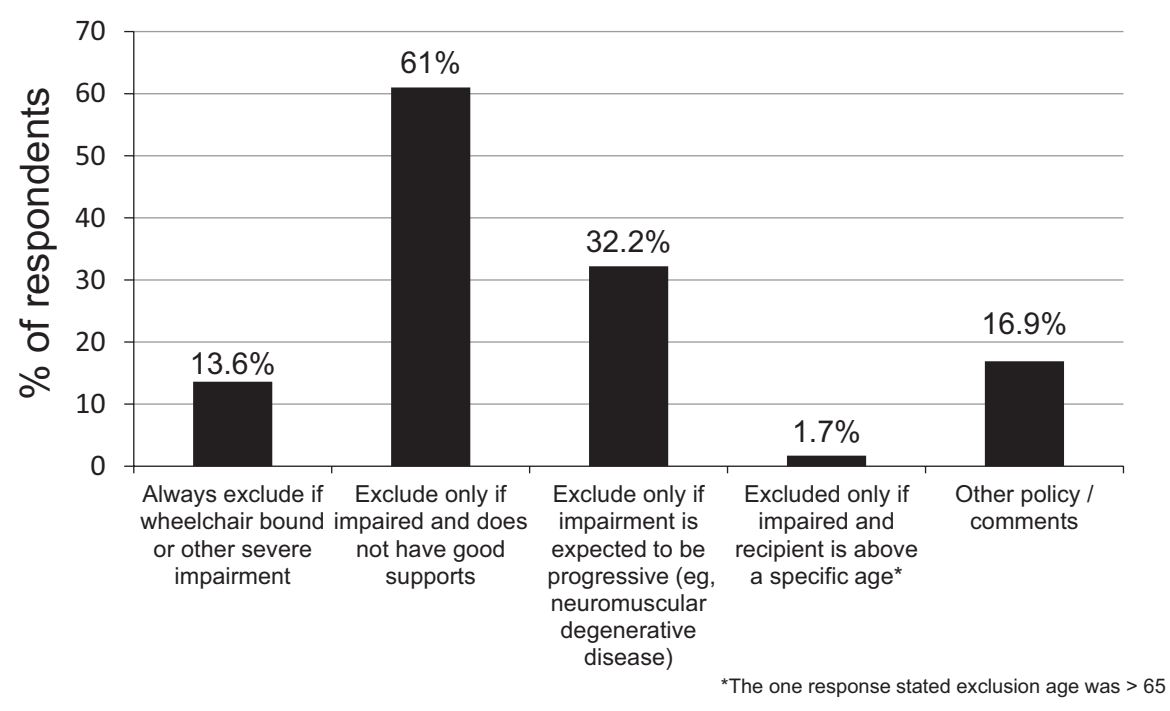

FIGURE 5 Does your program accept potential recipients with functional impairment (choose all that apply)? $\mathrm{n}=59$

\section{3 | Waitlist management}

Several additional questions were asked regarding waitlist management. Most programs $(88.1 \%, n=52)$ do not have an upper age cutoff for delisting a potential kidney transplant recipient who was previously listed. However, $8.5 \%(n=5)$ of respondents do report having an age cutoff for delisting, ranging from 75 to 85 , mean $79 \pm 4$.2. While delisting based on age is even less common than denying the initial listing based on age, the presence of various comorbidities is commonly cited as a reason to delist older candidates (Figure 6). The most common reported frequency of re-evaluation of waitlisted patients $(n=58)$ was yearly $(51.8 \%)$, with every two years (8.9\%) and every six months (7.1\%) being much less common practices. A substantial proportion (30.4\%) responded with "other policy," including comments that the re-evaluation frequency depends on comorbidities and distance from the top of the list, such that evaluations would be repeated when candidates approached the top.

When asked whether re-evaluations of waitlisted candidates are more frequent if they are older $(n=55), 60.0 \%$ of programs said no, while $36.4 \%$ responded yes, with an age cutoff ranging from 60 to 70 , mean $65.8 \pm 4$. Similarly, the frequency of repeat cardiac testing
( $n=58$ ) usually does not depend on age (65.5\%), but $29.3 \%$ do perform more frequent testing, using an age cutoff ranging from 50-70, mean $59.2 \pm 7.6$ years old. When asked "if a high quality kidney from a young deceased donor is available, will you ever skip an older potential recipient who is at the top of the list, to give the kidney to someone who is younger $(n=57)$, most respondents said no $(71.9 \%)$, but $12.3 \%$ said yes, with an age cutoff ranging from 60 to 70 , mean $65 \pm 7.1$ years old.

In addition to the above descriptive statistics, we analyzed the responses to see whether we could detect any patterns of different answers based on (i) respondent age, (ii) nephrology or surgical position, (iii) size of program, and (iv) higher or lower percentage of transplant recipients $\geq 65$ years old. We did not detect any statistically significant differences in answers based on any of these four respondent characteristics.

\section{4 | DISCUSSION}

In areas of medicine with high quality detailed data to guide decisionmaking, practices tend to be relatively uniform among different 
FIGURE 6 What other criteria does your program use to de-list older potential recipients (check all that apply)? $\mathrm{n}=59$

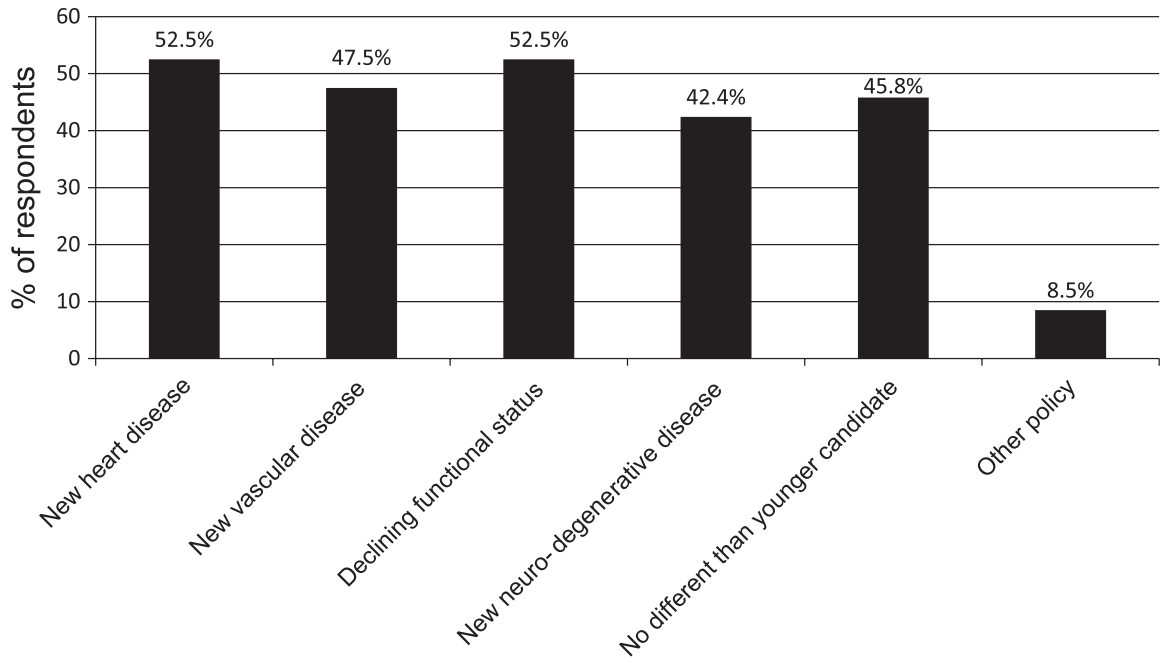

providers. However, in the absence of good data, practices vary significantly. Given the limited data to guide U.S. transplant centers in the evaluation and selection of older candidates in listing for kidney transplantation, it is not surprising for us to find that practices differ greatly between centers. The results of our survey demonstrate a wide range in the percentage of over 65 year olds receiving kidney transplants, from $3 \%$ to $50 \%$. These differences may partly reflect differences in local demographics, but also reflect widely different approaches to accepting these older patients.

In general, programs are hesitant to use absolute chronological cutoffs (76.3\% of programs), but if they decide to do so, they pick a relatively high cutoff (median 80 years old). This approach likely reflects that used in many other areas of medicine, in that "physiological" age is considered more important in predicting outcomes than chronological age. While we do not report any statistical analysis of the respondent's comments explaining their answers, this distinction between physiological and chronological age was the most frequent comment seen. Still others pointed out that they decide whether or not to list candidates on a "case by case basis," without strict protocols. Relatively few programs report protocols suggesting absolute age cutoffs for listing in the context of specific comorbidities such as cardiovascular disease (Figure 2). This overall approach of not excluding candidates purely on the basis of age is also consistent with the recommendations of recent reviews and guidelines. ${ }^{12}$

Almost one-third of U.S. programs may try to get at the distinction between physiological and chronological age by having listing guidelines based on life expectancy without transplant, but the reported life-expectancy cutoff varies from 2 to 10 years. Such an approach may be conceptually reasonable, but estimating life expectancy is challenging in itself. Interestingly, $61 \%$ of respondents said they would transplant patients even if the expected benefit would only be in quality, not length of life.

Only $25.4 \%$ of our respondents report performing functional assessments on all candidates, but most of the other programs will perform such assessments if an informal assessment suggests doing so. Yet, how to use this information continues to vary significantly between programs (Figure 5). Similarly, programs have variable ways of using the information that a candidate lives in a nursing home (Figure 3) or has cognitive impairment (Figure 4).

Some transplant providers have questioned the advisability of using live donors for older recipients. However, we find that the vast majority of U.S. programs would accept a live donor for an older recipient, even if that donor were a nondirected donor. In fact, in the comments section of the survey, many respondents reported that while they would list older candidates, they would often only do so if the candidate had a live donor, presumably because of poor expected recipient outcomes after prolonged listing for a deceased donor.

This study has all the limitations of any emailed survey. There is potential bias in self-reporting practices, although the answers were anonymous and it is hard to imagine any pressure on respondents to provide answers that are in any way "better" than the reality. There is also a potential for respondents' not being representative of all US transplant centers. We did find in the UNOS database the actual number of transplants performed in 2012, based on the respondent's reported 4 digit UNOS code and calculated a mean number of transplants of 99.5 , somewhat lower than the reported mean of 108.5. However, the mean number of transplants performed in nonpediatric programs in the USA in 2012 was 77.2, ${ }^{1}$ not statistically different from the 99.5 mean of respondent programs $(P=.11)$. This suggests that, at least based on program size, our respondents were similar to all U.S. programs. We also note that those who were 65 or over comprised $18.4 \%$ of patients transplanted in $2012,{ }^{1}$ not far from the mean $22.9 \%$ of our respondents' reported waitlist. As we did not survey pediatric programs, it is not surprising that the national data, which includes pediatric programs, reports a somewhat lower percentage of $\geq 65$-year-old recipients. Detailed information on the percentage of $\geq 65$-year-old transplants in nonpediatric programs is not available to make a direct comparison to our respondents.

Our findings of variable practice patterns in particular areas, such as the impact of cognitive or functional impairments, highlight some specific areas that may be particularly fruitful for research. For example, the difficulty in estimating physiological age remains, but it may be that formal measurements of frailty ${ }^{13}$ will be helpful additions in that assessment. Clearly, further studies are needed regarding the 
impact of age and comorbidities on kidney transplant outcomes, and on which older patients are most appropriate for transplant listing.

\section{CONFLICT OF INTEREST}

The authors declare no conflicts of interest.

\section{AUTHORS' CONTRIBUTIONS}

Didier A. Mandelbrot: Conceived and designed project, designed survey tool, analyzed data, and wrote the manuscript; Aaron Fleishman: Designed survey tool, collated and analyzed data, and revised the manuscript; James R. Rodrigue and Silas P. Norman: Designed survey tool, analyzed data, revised the manuscript; Millagra Samaniego: Conceived and designed project, designed survey tool, analyzed data, and revised the manuscript.

\section{ORCID}

Didier A. Mandelbrot iD http://orcid.org/0000-0003-3326-8583

James R. Rodrigue (iD http://orcid.org/0000-0001-5589-4919

\section{REFERENCES}

1. OPTN/SRTR 2012. Annual Data Report. 2014

2. Schaeffner ES, Rose C, Gill JS. Access to kidney transplantation among the elderly in the United States: a glass half full, not half empty. Clin J Am Soc Nephrol. 2010;5:2109-2114.

3. Lloveras J, Arcos E, Comas J, Crespo M, Pascual J. A paired survival analysis comparing hemodialysis and kidney transplantation from deceased elderly donors older than 65 years. Transplantation. 2015;99:991-996.

4. Fabrizii V, Winkelmayer WC, Klauser R, et al. Patient and graft survival in older kidney transplant recipients: does age matter? J Am Soc Nephrol. 2004;15:1052-1060.
5. Rao PS, Merion RM, Ashby VB, Port FK, Wolfe RA, Kayler LK. Renal transplantation in elderly patients older than 70 years of age: results from the Scientific Registry of Transplant Recipients. Transplantation. 2007;83:1069-1074.

6. Gill JS, Schaeffner E, Chadban S, et al. Quantification of the early risk of death in elderly kidney transplant recipients. Am J Transplant. 2013;13:427-432.

7. Heldal K, Hartmann A, Grootendorst DC, et al. Benefit of kidney transplantation beyond 70 years of age. Nephrol Dial Transplant. 2010;25:1680-1687.

8. Veroux M, Grosso G, Corona D, et al. Age is an important predictor of kidney transplantation outcome. Nephrol Dial Transplant. 2012;27:1663-1671.

9. Grams ME, Kucirka LM, Hanrahan CF, Montgomery RA, Massie AB, Segev DL. Candidacy for kidney transplantation of older adults. J Am Geriatr Soc. 2012;60:1-7.

10. Faravardeh A, Eickhoff M, Jackson S, et al. Predictors of graft failure and death in elderly kidney transplant recipients. Transplantation. 2013;96:1089-1096.

11. Abecassis M, Bridges ND, Clancy CJ, et al. Solid-organ transplantation in older adults: current status and future research. Am J Transplant. 2012;12:2608-2622.

12. Segall L, Nistor I, Pascual J, et al. Criteria for and appropriateness of renal transplantation in elderly patients with end-stage renal disease: a literature review and position statement on behalf of the European Renal Association-European Dialysis and Transplant Association Descartes Working Group and European Renal Best Practice. Transplantation. 2016;100:e55-e65.

13. McAdams-DeMarco MA, Tan J, Salter ML, et al. Frailty and cognitive function in incident hemodialysis patients. Clin J Am Soc Nephrol. 2015;10:2181-2189.

How to cite this article: Mandelbrot DA, Fleishman A, Rodrigue JR, Norman SP, Samaniego M. Practices in the evaluation of potential kidney transplant recipients who are elderly: A survey of U.S. transplant centers. Clin Transplant. 2017;31:e13088. https://doi.org/10.1111/ctr.13088 